Polymer Journal, Vol. 38, No. 8, pp. 819-826 (2006)

(C) 2006 The Society of Polymer Science, Japan

\title{
Two Different Surface Properties of Regenerated Cellulose due to Structural Anisotropy
}

\author{
Chihiro Yamane, ${ }^{1, \dagger}$ Takeshi Aoyagi, ${ }^{2}$ Mariko Ago, ${ }^{3}$ Kazuishi SAto, ${ }^{3}$ \\ Kunihiko OKAJIMA, ${ }^{3}$ and Toshisada TAKAHASHI ${ }^{1}$ \\ ${ }^{1}$ Faculty of Home Economics, Kobe Women's University, Kobe 654-8585, Japan \\ ${ }^{2}$ Central Research Laboratory, Asahi Kasei Corporation, Shizuoka 416-8501, Japan \\ ${ }^{3}$ Faculty of Engineering, Tokushima Bunri University, Kagawa 769-2193, Japan
}

(Received December 7, 2005; Accepted April 20, 2006; Published July 7, 2006)

\begin{abstract}
Contact angles of water droplet on regenerated cellulose films as an index of wettability were positively correlated with the orientation of (1-10) crystal planes and crystallinity. Because hydroxyl groups of cellulose are located at the equatorial positions of glucopyranose rings, corresponding to the surface of (1-10) crystal planes, the hydrophilicity of the (1-10) surface is expected to be very high. It is natural, therefore, that higher planar orientation of (1-10) planes and crystallinity lead to higher density of hydroxyl groups on the surface of regenerated cellulose films resulting in higher wettability. In contrast, hydrogen atoms are located at the axial positions of the glucopyranose rings, corresponding to the surface of (110) planes. Thus, the (110) surface is expected to be hydrophobic, and the surface energy obtained by computer simulations was far lower than that of the (1-10) surface. This suggests that cellulose with complementary properties, i.e., hydrophobicity, may be created by structural controls such as reversing the planar orientation from (1-10) to (110). Although it was not possible to reverse this orientation completely, post-treatments with a nonpolar solvent (e.g., hexane), liquid ammonia or hot glycerol can somewhat control the wettability of regenerated cellulose films. [doi:10.1295/polymj.PJ2005187]

KEY WORDS Cellulose / Wettability / Contact Angle / Hydrophilic Surface / Hydrophobic Surface / Planar Orientation /
\end{abstract}

Regenerated cellulose is known to be one of the most hydrophilic polymers. The contact angle of water droplet on typical regenerated cellulose films, such as cellophane and cuprophane, is $c a .12^{\circ}$, far lower than those of widely used polymers such as poly(vinyl alcohol) (PVA), 36 ; poly(methyl methacrylate), 57; poly(vinyl acetate), $63^{\circ}$; nylon, $70^{\circ}$; poly(vinylidene chloride), $80^{\circ}$; poly(vinyl chloride), $87^{\circ}$; poly(styrene), $91^{\circ}$; poly(ethylene), $94^{\circ}$; poly(propylene), $95^{\circ}$; and poly(tetrafluoroethylene), $108^{\circ} .1$ Although the hydrophilic polymer poly(vinyl alcohol), having many hydroxyl groups on the molecule, has a lower contact angle $\left(36^{\circ}\right)$ than the other polymers listed above, this value is still higher than that of regenerated cellulose films. It is also worthy to note that the contact angle of water on starch films is $41^{\circ}$. Why is cellulose more wettable than PVA, which is water soluble; and starch, which is also water soluble and even has the same molecular formula as cellulose? It has been reported that the high wettability of cellulose films results from the higher density of hydroxyl groups on a cellulose surface than on a PVA surface, ${ }^{2}$ but this explanation has never been discussed from a structural point of view.

Conversely, many experimental facts show that cellulose interacts strongly with hydrophobic (nonpolar) organic solvents. For example, hexane, toluene and dichloromethane are captured in cellulose even after extensive vacuum drying, ${ }^{3}$ paraffin is easily dispersed in water with a small amount of cellulose particles, ${ }^{4}$ and relaxation peaks of dynamic viscoelastic measurements or thermally stimulated depolarized current measurements on cellulose shift to lower temperature in nonpolar solvents. ${ }^{5,6}$ These findings suggest that cellulose has a hydrophobic nature as well.

In this paper, we discuss the essence of the hydrophilic nature of cellulose by focusing on structural anisotropy, and then try to clarify its hydrophobic nature also resulting inevitably from structural anisotropy.

\section{EXPERIMENTAL}

\section{Sample Preparations}

Cuprophane. Cotton linter with viscosity-average molecular weight $M_{\mathrm{v}}=1.7 \times 10^{5}$ was dissolved in aqueous cuprammonium hydroxide solution according to a known procedure ${ }^{7}$ at a cellulose concentration of $8 \mathrm{wt} \% . M_{\mathrm{v}}$ was estimated by using eq 1 , established for cellulose/cadoxen systems. ${ }^{8}$

$$
[\eta]=3.85 \times 10^{-2} M_{\mathrm{v}}^{0.76}
$$


The cellulose solution was cast on a glass plate to give a thickness of $500 \mu \mathrm{m}$, which was immersed gently in water for $1 \mathrm{~min}$ at $25^{\circ} \mathrm{C}$ and regenerated by $2 \mathrm{wt} \%$ aqueous sulfuric acid for $10 \mathrm{~min}$, followed by washing with water and drying in air with a given dimension.

Cellophane. A softwood sulfite pulp mainly from white spruce (ALAPUL-T, Alaska Pulp Co., Ltd.) was immersed in an $18 \mathrm{wt} \%$ aqueous $\mathrm{NaOH}$ solution at $25^{\circ} \mathrm{C}$. An excess liquid was squeezed out until the cellulose concentration reached $30 \mathrm{wt} \%$. The squeezed pulp was hydrolyzed in air to give a $M_{\mathrm{v}}=4.9 \times$ $10^{4}$, and this was followed by reaction with carbon disulfide. Next, a dilute aqueous $\mathrm{NaOH}$ solution was poured into the wet pulp with mixing in a kneader to prepare a cellulose solution with the following composition: $8.5 \mathrm{wt} \%$ cellulose, $6.0 \mathrm{wt} \% \mathrm{NaOH}$, and degree of $\mathrm{CS}_{2}$ substitution of 0.45 . This solution was cast on a glass plate to give a thickness of $500 \mu \mathrm{m}$. These were immersed gently into a coagulation bath which contained $14 \mathrm{wt} \% \mathrm{H}_{2} \mathrm{SO}_{4}, 26 \mathrm{wt} \% \mathrm{Na}_{2} \mathrm{SO}_{4}$, and $1.5 \mathrm{wt} \% \mathrm{ZnSO}_{4}$, at $50{ }^{\circ} \mathrm{C}$ for $5 \mathrm{~min}$. Finally, the films were washed with water and dried in air with a given dimension.

Films from Cellulose/Aqueous $\mathrm{NaOH}$ Solution. The sulfite pulp used above was subjected to steam explosion treatment and the resultant refined exploded pulp with $M_{\mathrm{v}}=5.2 \times 10^{4}$ was dissolved in aqueous $\mathrm{NaOH}$ according to a previously published procedure. ${ }^{5}$ The solution with a cellulose concentration of $5 \mathrm{wt} \%$ and $\mathrm{NaOH}$ concentration of $7.6 \mathrm{wt} \%$ was cast on a glass plate to give a thickness of $500 \mu \mathrm{m}$. This was immersed gently in aqueous sulfuric acid with various concentrations ranging from $20 \mathrm{wt} \%$ to 65 wt $\%$ at $-7^{\circ} \mathrm{C}$ for $5 \mathrm{~min}$, followed by washing with water and drying in air with a given dimension. Thereafter, these films were designated with the abbreviation SK-14.

Films from Cellulose/DMAc-LiCl Solution. The same cotton linter used for the cuprophane preparation was dissolved at a cellulose concentration of $3 \mathrm{wt} \%$ in dimethylacetamide (DMAc) containing $8 \mathrm{wt} \%$ of lithium chloride ( $\mathrm{LiCl})$, according to the procedure described by Turbak et al. ${ }^{9}$ The cellulose/DMAc-LiCl solution was cast on a glass plate to give a thickness of $500 \mu \mathrm{m}$. This film was then immersed gently in acetone and toluene for $1 \mathrm{~min}$ each at $25^{\circ} \mathrm{C}$, followed by washing with methanol and drying in air with a given dimension.

Micro-Crystalline Cellulose Films. The regenerated cellulose from cuprammonium solution (Bemlise ${ }^{\circledR}$ : Asahi Kasei Co., Ltd; $M_{\mathrm{v}}=1.3 \times 10^{5}$ ) was hydrolyzed by $50 \mathrm{wt} \%$ aqueous $\mathrm{H}_{2} \mathrm{SO}_{4}$ at $70^{\circ} \mathrm{C}$ for $16 \mathrm{~h}$. This removes the amorphous regions of cellulose and greatly increases crystallinity. This micro-crystalline cellulose was washed repeatedly with water by decantation of supernatant, resulting from centrifugation at $10,000 \mathrm{rpm}$ for $10 \mathrm{~min}$. The micro-crystalline cellulose suspension was cast on a glass plate to give $500 \mu \mathrm{m}$ thick films, which were dried in air. Thereafter, these films were designated micro-crystalline cellulose films.

Amorphous Cellulose Films. The same sulfite pulp used for the cellophane preparation was dissolved in $65 \mathrm{wt} \%$ sulfuric acid solution at $0{ }^{\circ} \mathrm{C}$, giving a dope with cellulose concentration of $4 \mathrm{wt} \%$. The solution was poured into water at $0{ }^{\circ} \mathrm{C}$ to precipitate amorphous cellulose, ${ }^{4}$ which was hydrolyzed using a 20 wt $\%$ sulfuric acid solution at $80^{\circ} \mathrm{C}$ for $20 \mathrm{~min}$ and then washed with water. A $1.5 \mathrm{wt} \%$ cellulose suspension was pulverized into sub-micron particles with an ultra high pressure homogenizer (Microfluidizer ${ }^{\mathrm{TM}}$ M-110EM, Mizuho Kogyo Co., Ltd., Japan). ${ }^{4}$ This pulverized cellulose suspension was cast on a glass plate to give a thickness of $500 \mu \mathrm{m}$ and dried in air. Thereafter, these films were designated amorphous cellulose films.

\section{Post-Treatments of Cellulose Films}

Cellophane was treated with the following liquids under the given conditions: cyclohexane at $25^{\circ} \mathrm{C}$ for $24 \mathrm{~h}$, liquid ammonia at $-80^{\circ} \mathrm{C}$ for $5 \mathrm{~min}$, and hot glycerin at $260^{\circ} \mathrm{C}$ for $7 \mathrm{~min}$. The latter two treatments changed the cellulose polymorph from Cell II to Cell III and Cell IV, respectively. Completion of these crystal transitions were confirmed by wide angle $\mathrm{X}$-ray diffraction patterns.

\section{Planar Orientation}

$\mathrm{X}$-ray diffraction patterns of samples were measured by the reflection method and recorded on an Xray diffractometer with scintillation counter (Rotaflex Ru-200PL, Rigaku Denki Co., Ltd., Japan). Samples were irradiated at $2 \theta=5-35^{\circ}$ to the membrane surface (parallel incidence), and measurements were made at $1^{\circ} / \mathrm{min}$ with an internal reference $\left(\mathrm{SiO}_{2} ; 2 \theta=\right.$ $28.45^{\circ}$ ). To calculate the orientation of (1-10) crystal planes, diffraction intensities of parallel incidence $\left(\mathrm{I}_{(1-10)}\right)$ at $2 \theta=c a \cdot 12^{\circ},\left(\mathrm{I}_{(110)}\right)$ at $2 \theta=c a \cdot 20^{\circ}$ and $\left(\mathrm{I}_{(020)}\right)$ at $2 \theta=c a .22^{\circ}$ were measured. X-ray diffraction patterns of randomly oriented samples were recorded by the powder method. In order to prepare the randomly oriented samples, films were cut into particle-like size to ameliorate the influence of crystalline orientation (e.g., uni-planar orientation). Diffraction intensities of the randomly oriented samples (i) of each crystal plane were measured. It is well known that the (1-10) crystal plane is oriented parallel to the film surface in almost all regenerated cellulose films. The orientation index, $f(1-10)$ for the (1-10) plane was evaluated by the simple method reported by 
Takahashi, ${ }^{10}$ using the eq 2 . The orientation index provides a relative degree of planar orientation and takes on values from 0 (random) to 1 (parallel to surface).

$$
f_{(1-10)}=\frac{I_{(1-10)}-I_{(110)+(200)} \times \frac{i_{(1-10)}}{i_{(110)+(200)}}}{I_{(1-10)}}
$$

\section{Crystallinity}

The X-ray diffraction patterns of the randomly oriented samples were used for calculating crystallinity index $X_{c} \cdot X_{c}$ was estimated from peak areas responsible for the (1-10), (110), and (020) planes, separated by the Lorentz-Gaussian peak separation method.

\section{Contact Angle}

Contact angles were measured by taking photographs of small droplets of water on film samples using a contact angle meter (CA-A, Kyowa Interface Science Co., Ltd). More than five readings on different droplets from the same sample were averaged. The deviation of each reading from the average was within $\pm 1^{\circ}$. It is well known that surface morphology greatly affects contact angle. Wenzel's equation, $\cos \theta^{\prime}=r \cos \theta$, relates the effect of surface area to the contact angle, where $\theta^{\prime}, \theta$ and $\mathrm{r}$ are contact angle of uneven surface, contact angle of smooth surface and ratio of uneven/smooth surface area, respectively. ${ }^{11}$ According to the equation, $\theta^{\prime}$ is smaller than $\theta$ at $\theta<90^{\circ}$ as in the case of cellulose. However, because we found all samples to be almost flat and to have the same structures (by SEM observations in the $500 \mathrm{X}$ to $10000 \mathrm{X}$ magnification range), we ignored morphology effects here.

The material which contacts a polymer during the film preparation process affects the properties of the polymer surface. ${ }^{12,13}$ In order to avoid this effect, we used the film surface which was opposite to the side contacting the glass plate during film preparation. Immediately prior to measurement, the films were re-dried under vacuum at $70^{\circ} \mathrm{C}$ for $12 \mathrm{~h}$.

\section{Viscoelastic Measurements}

The viscoelastic properties (mechanical loss tangent $\delta, \tan \delta$ - temperature T curves) of the cellulose films were recorded on a viscoelastic spectrometer (Model SDM-5000, Seiko Denshi Co. Ltd., Japan) under the following conditions: frequency of $10 \mathrm{~Hz}$, heating rate of $10^{\circ} \mathrm{C} / \mathrm{min}$, measuring interval of $1{ }^{\circ} \mathrm{C} /$ min, sample length of $20 \mathrm{~mm}$, sample width of $5 \mathrm{~mm}$, initial charge of $10 \mathrm{~g} / \mathrm{mm}^{2}$, and temperature range of $-150-350^{\circ} \mathrm{C}$.

\section{Calculation of Surface Energy}

Crystal models of Cell $\mathrm{I}_{\alpha}$, Cell $\mathrm{I}_{\beta}$ and Cell II were constructed by using atomic coordinates reported by Nishiyama et al. ${ }^{14-16}$ Each crystal models contained 192 glucose residues and had $20-40 \AA$ in length of each sides. Vacuum slab was attached on the crystal plane, of which surface energy was to be calculated, for the crystal models with crystal surface. Free energy $G_{\text {surface }}$ and $G_{\text {bulk }}$ of the crystal models with and without the crystal surface, respectively, were obtained from molecular mechanics calculations with COMPASS force-field parameters using Forcite software (Accelrys software Inc.). Let A denote the surface area of the former crystal model. The surface energy $\gamma$ of the crystal plane for cellulose polymorphs (Cell $\mathrm{I}_{\alpha}$, Cell $\mathrm{I}_{\beta}$, Cell II) was calculated from the following eq 3 . Nonbonding energy was cut off by $12.5 \AA$, Coulomb force was calculated with the Ewald method.

$$
\gamma=\frac{\mathrm{G}_{\text {surface }}-\mathrm{G}_{\text {bulk }}}{A}
$$

\section{RESULTS AND DISCUSSION}

\section{Effect of Structural Parameters on Wettability of Film Surfaces}

Figure 1 shows X-ray diffraction patterns of the film sample and the randomly oriented sample from cellulose/aqueous $\mathrm{NaOH}$ solution followed by the coagulation of $40 \% \mathrm{H}_{2} \mathrm{SO}_{4}$ (SK-14 $40 \%$ in Table I). The randomly oriented sample was prepared by cutting of the film sample into particle-like size in order to ameliorate the influence of crystalline orientation (e.g., uni-planar orientation). An intensity of (1-10) crystal plane, $\mathrm{I}_{(1-10)}$, of the film sample was much higher than that of the randomly oriented sample. This means that the (1-10) crystal plane is oriented parallel to the film

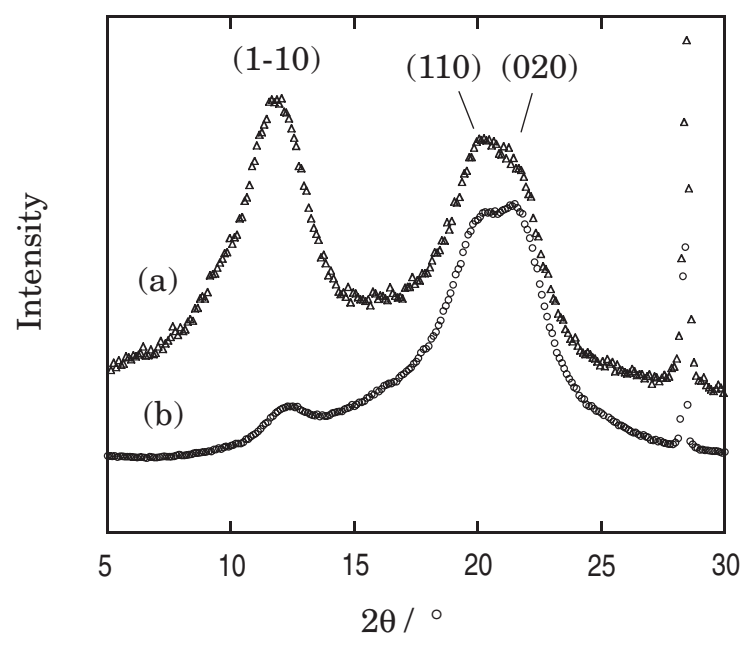

Figure 1. X-Ray diffraction patterns of the film sample (a) and the randomly oriented sample (b) from cellulose/aqueous $\mathrm{NaOH}$ solution followed by the coagulation of $40 \% \mathrm{H}_{2} \mathrm{SO}_{4}$. 
Table I. Contact angles and structural parameters of cellulose films

\begin{tabular}{|c|c|c|c|c|c|c|c|c|c|c|c|c|}
\hline \multirow{2}{*}{\multicolumn{2}{|c|}{ Sample }} & \multirow{2}{*}{$\begin{array}{c}\text { Contact } \\
\text { angle } \\
\text { /deg. }\end{array}$} & \multirow[b]{2}{*}{$\mathrm{f}(1-10)$} & \multirow[b]{2}{*}{$\begin{array}{l}X_{c} \\
/ \%\end{array}$} & \multicolumn{2}{|c|}{$\beta_{2}$} & \multicolumn{2}{|c|}{$\beta_{1}$} & \multicolumn{2}{|c|}{$\alpha_{\mathrm{sh}}$} & \multicolumn{2}{|c|}{$\alpha_{1}$} \\
\hline & & & & & $\begin{array}{l}\mathrm{T}_{\max } \\
/{ }^{\circ} \mathrm{C}\end{array}$ & $\tan \delta_{\max }$ & $\begin{array}{l}\mathrm{T}_{\max } \\
/{ }^{\circ} \mathrm{C}\end{array}$ & $\tan \delta_{\max }$ & $\begin{array}{l}\mathrm{T}_{\max } \\
/{ }^{\circ} \mathrm{C}\end{array}$ & $\tan \delta_{\max }$ & $\begin{array}{l}\mathrm{T}_{\max } \\
/{ }^{\circ} \mathrm{C}\end{array}$ & $\tan \delta_{\max }$ \\
\hline \multirow{2}{*}{\multicolumn{2}{|c|}{$\begin{array}{l}\text { Cupro. } \\
\text { Viscose }\end{array}$}} & 12.2 & 0.52 & 42 & -75.9 & 0.0442 & -61.9 & 0.0400 & 169.0 & 0.0660 & 290.0 & 0.0900 \\
\hline & & 11.6 & 0.60 & 30 & -62.3 & 0.0321 & -40.9 & 0.0300 & - & - & 286.7 & 0.0816 \\
\hline \multirow[t]{4}{*}{ SK-14 } & $20 \%{ }^{\mathrm{a}}$ & 10.5 & 0.67 & 45 & -88.5 & 0.030 & - & - & 218.8 & 0.0672 & 289.1 & 0.0734 \\
\hline & $40 \%^{\mathrm{a}}$ & 16.7 & 0.60 & 27 & -84 & 0.036 & - & - & 228.1 & 0.0714 & 282.5 & 0.0760 \\
\hline & $50 \%^{\mathrm{a}}$ & 19.3 & 0.52 & 23 & -83.0 & 0.037 & - & - & 232.8 & 0.0740 & 279.7 & 0.0802 \\
\hline & $60 \%^{a}$ & 21.8 & 0.08 & 20 & -78.5 & 0.038 & - & - & 237.5 & 0.0771 & 277.3 & 0.0844 \\
\hline \multicolumn{2}{|c|}{ DMAc/LiCl } & 40.5 & 0.09 & 6 & -70.9 & 0.0360 & -60.6 & 0.0390 & 155.0 & 0.0560 & 272.0 & 0.0670 \\
\hline \multicolumn{2}{|c|}{$\begin{array}{l}\text { Micro-Crystal- } \\
\text { line Cellulose }\end{array}$} & 11.5 & 0.75 & 78 & - & - & - & - & - & - & - & - \\
\hline \multicolumn{2}{|c|}{ Amorphous } & 42.0 & - & 0 & - & - & - & - & - & - & - & - \\
\hline
\end{tabular}

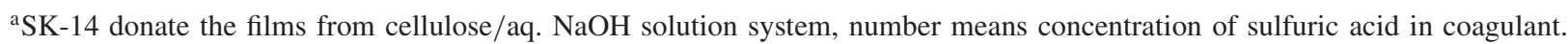

surface. This kind of the orientation is generally known in almost all regenerated cellulose films. ${ }^{10}$ The planar orientation index $\mathrm{f}(1-10)$ was calculated from the eq 2 comparing $\mathrm{I}_{(1-10)}$ and $\mathrm{I}_{(110)+(020)}$ of both film samples and randomly oriented samples. Crystallinity index $\mathrm{X}_{\mathrm{c}}$ was estimated from the $\mathrm{X}$-ray diffraction patterns of randomly oriented samples. The diffraction peaks at $2 \theta=28.45^{\circ}$ is from $\mathrm{SiO}_{2}$ as an internal reference.

Table I shows contact angles of water droplet on regenerated cellulose films with various structural parameters. The contact angles tended to be low when $f(1-10)$ and $X_{c}$ were high. The sample with the highest contact angle, $42^{\circ}$, was observed for the amorphous cellulose film. Because of its amorphous structure, $\mathrm{f}(1-10)$ could not be determined for this material. It is noteworthy that this contact angle was nearly the same as that of starch, $41^{\circ}$.

Figure 2 shows the relationship between $\mathrm{f}(1-10)$ and water contact angles. Numerals in the figure indicate $X_{c}$. The higher the $f(1-10)$ was, the lower the contact angle became. In addition, high $\mathrm{X}_{\mathrm{c}}$ values yielded significantly lower contact angles. Specifically, the contact angle of Cuprophane, $X_{c}=42 \%$, was lower than that of SK-14 50\% with $X_{c}=23 \%$, whereas both had the same value of $f(1-10)$, namely 0.52 . Contrary to popular belief that the hydrophilicity of amorphous form (which has high water absorbency) is much higher than that of the crystalline form, the wettability of regenerated cellulose, from the viewpoint of the surface, probably depends on its crystalline properties, as indicated by the $f(1-10)$ and $X_{c}$ values.

Table I also shows parameters for amorphous region of cellulose, namely mechanical absorption temperature $\mathrm{T}_{\max }$, absorption intensity $\tan \delta$, and water contact angles. Dynamic absorptions of regenerated cellulose are attributed to segmental motions as fol-

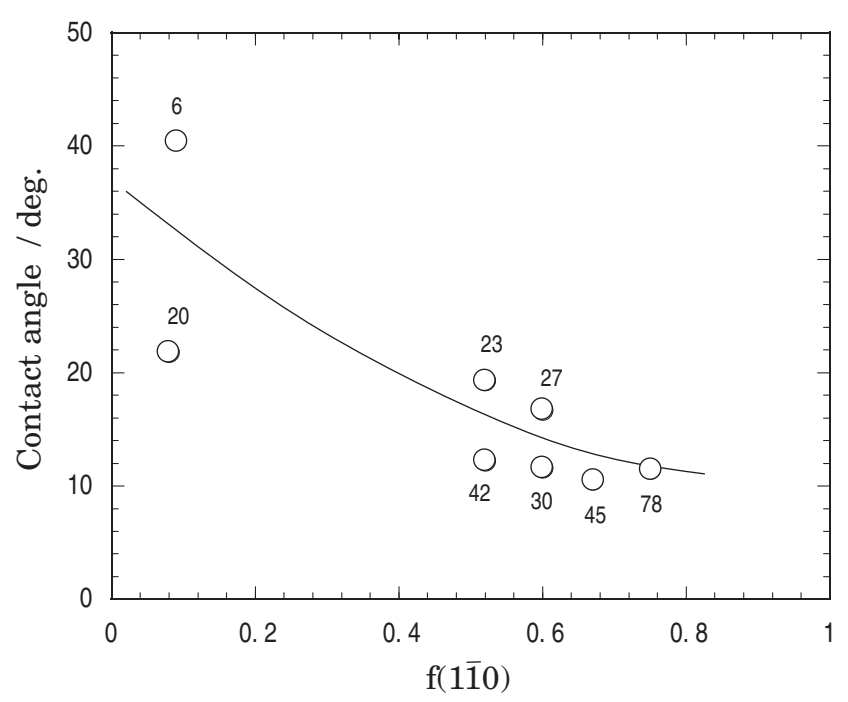

Figure 2. Relation between (1-10) plane orientation index $\mathrm{f}(1-10)$ and contact angle for water. Numbers denote crystallinity $\mathrm{X}_{\mathrm{c}}$.

lows: $\alpha$ for the micro-Brownian motions of cellulose main chain segments, and $\beta$ for the local twisting motions of main chains. The $\alpha$ and $\beta$ absorptions consist of $\alpha_{1}$ and $\alpha_{\mathrm{sh}}$ and $\beta_{1}$ and $\beta_{2}$ components, respectively. $\alpha_{\mathrm{sh}}$ is observed as a shoulder or maximum peak at a lower temperature than that of the $\alpha_{1}$ absorption peak. Here, $\alpha_{1}$ is assigned to the regions where intra- and intermolecular hydrogen bonds are highly developed. Some of the molecular chains in this region are immobile, as in the crystalline region. ${ }^{17}$ Because precise peaks for $\beta_{1}$ absorptions in the SK-14 series could not be detected, the effect of $\beta_{1}$ on water contact angles is not discussed here. Except for $\mathrm{T}_{\max } \alpha_{1}$, there were no relationships between amorphous parameters and water contact angles, as shown in Table I. Figure 3 shows the effect of $\mathrm{T}_{\max } \alpha_{1}$ on water contact angle. In this case, the water contact angles decreased with 


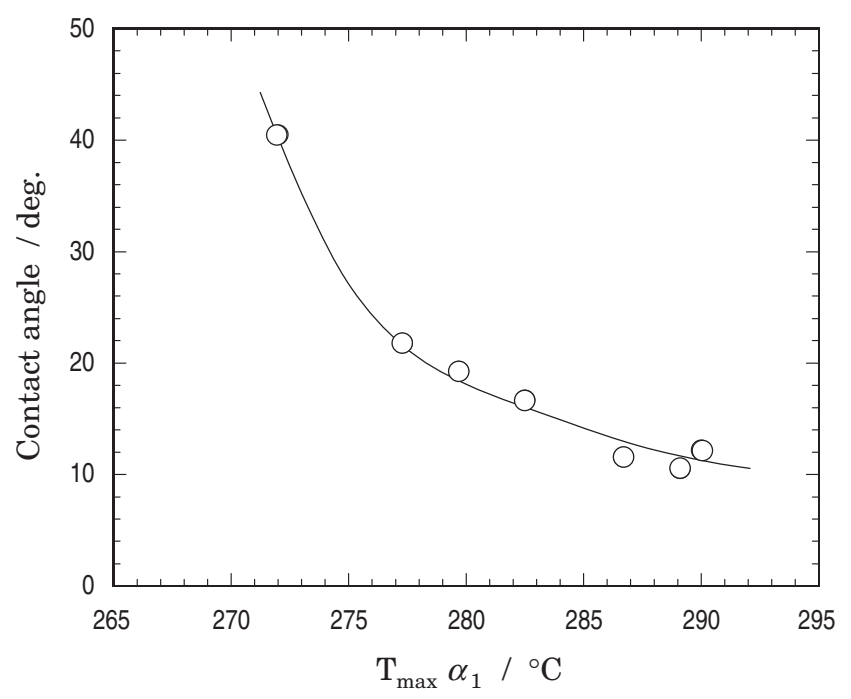

Figure 3. Relation between relaxation peak temperature for $\alpha_{1}\left(\mathrm{~T}_{\max } \alpha_{1}\right)$ and contact angle for water.

increasing $\mathrm{T}_{\max } \alpha_{1}$, as with the relation to $\mathrm{X}_{\mathrm{c}}$ shown in Figure 2. Though the structural region described by $\alpha_{1}$ is amorphous, the region is considered to have a crystal-like structure. When $\mathrm{T}_{\max } \alpha_{1}$ is high, activation energy of molecular relaxation and molecular density of the region are also high. This means that the higher $\mathrm{T}_{\max } \alpha_{1}$ probably makes the region more similar to the crystalline structure. Therefore, it is natural that $\mathrm{T}_{\max } \alpha_{1}$ had the same relation to water contact angles as did for $X_{c}$. The question was, why did the crystalline or crystal-like regions affect wettability so much more than the amorphous regions?

Figure 4 is a schematic representation of cellulose molecules with their hydrophilic and hydrophobic parts. ${ }^{18}$ The equatorial direction of the glucopyranose ring is hydrophilic because all three hydroxyl groups on the ring are located on the equatorial positions of the ring. In contrast, the axial direction of the ring is hydrophobic because of hydrogen atoms of $\mathrm{C}-\mathrm{H}$ bonds being located on the axial positions of the ring. Thus, cellulose molecules have intrinsically structural anisotropy. If these hydrophilic parts were arranged together in the same direction, a super hydrophilic surface with many hydroxyl groups would be formed. Figure 5 shows the unit cell of a cellulose II crystal and a plane structure model. The planar structure, which is closely related to the (1-10) crystal plane and formed by hydrophobic interactions between glucopyranose rings, has a hydrophilic surface. The glucopyranose rings in the (1-10) crystal plane are stacked with each other by hydrophobic interactions (such as van der Waals forces) and located perpendicular to the (1-10) surface. As the result, the density of hydroxyl groups on the (1-10) surface is very high, resulting in a hydrophilic surface. Because of the structural anisotropy of crystalline cellulose, higher $X_{c}$ and $f(1-10)$ values result in a larger surface area fraction of (1-10) planes in regenerated cellulose films, producing the most hydrophilic polymers, being more wettable than PVA or starch, as described above. As mentioned before, there is no relationship between amorphous parameters, except $\alpha_{1}$, and water contact angle. It is understandable that isotropic structures such as $\alpha_{\mathrm{sh}}$ or $\beta_{2}$ regions, have only a minor effect on wettability.

It is widely believed that surface roughness greatly affects water contact angles. ${ }^{11}$ We recognize the importance of surface morphology. However, the study of bulk structure is also important as a basis for understanding surface phenomena. In addition, we have al-

(a)
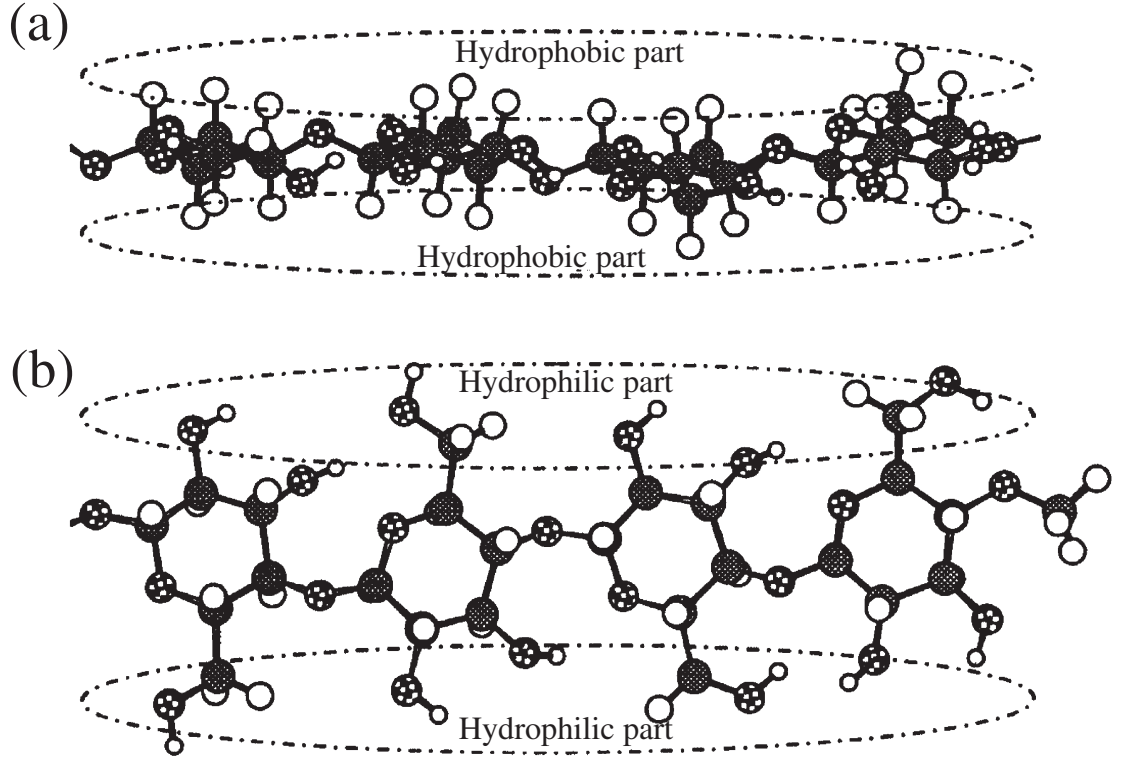

Figure 4. Hydrophobic and hydrophilic part of cellulose molecule: (a), end view of glucopyranose ring plane; (b), front view of glucopyranose ring plane. 


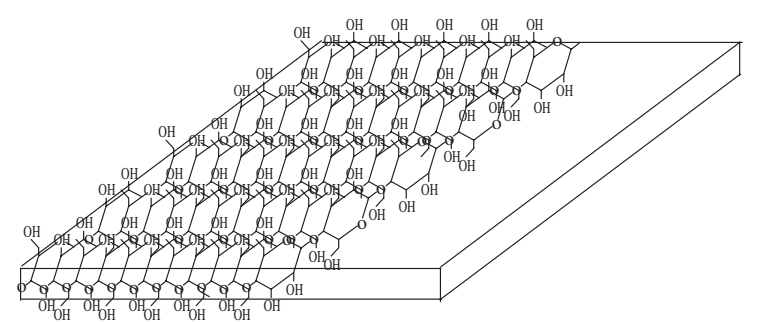

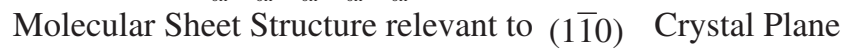

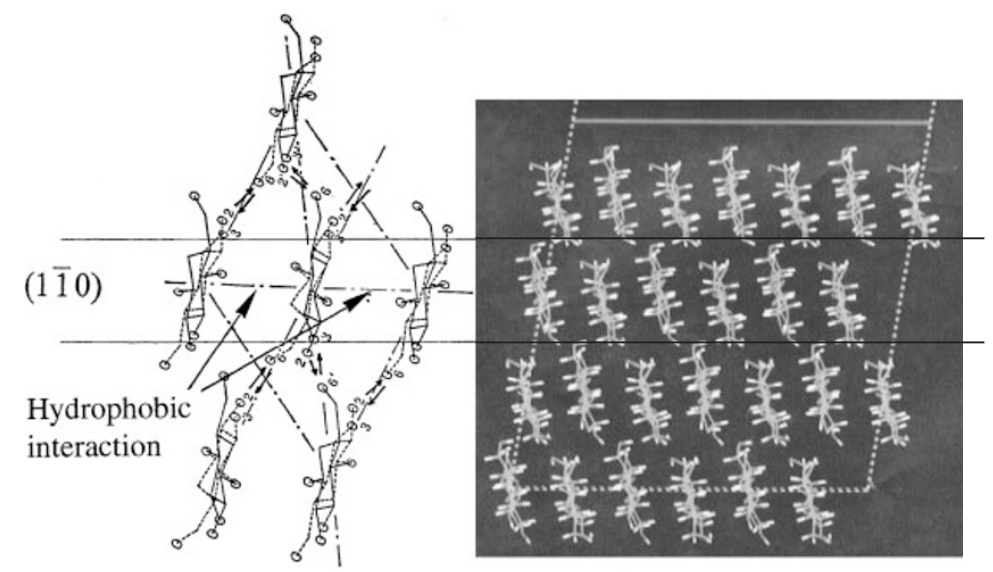

Figure 5. Schematic representation of unit cell of cellulose II and molecular sheet structure.

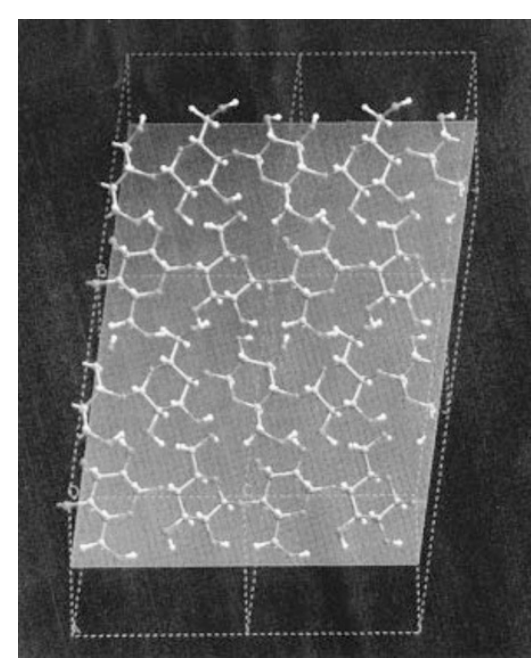

Over view of (110) crystal plane
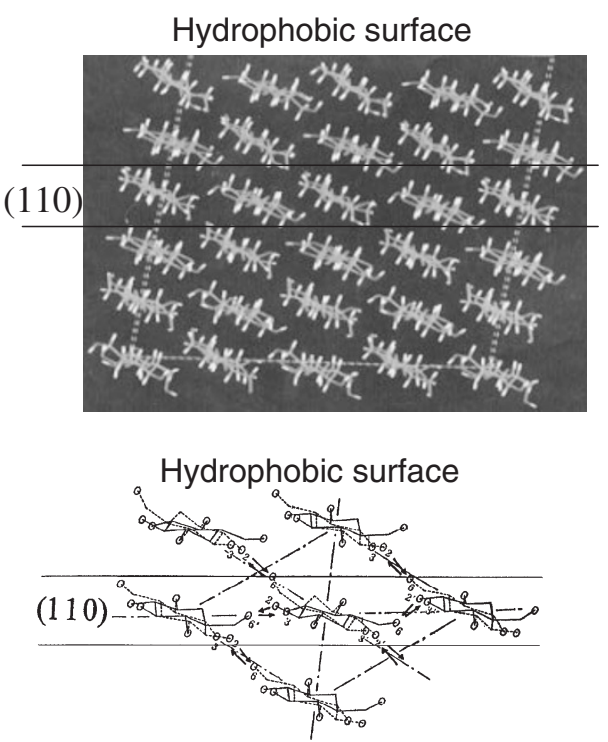

Figure 6. Schematic representation of hydrophobic surface in cellulose II.

so observed our film surfaces by SEM in the range from 500X to $10000 \mathrm{X}$ magnification, and found our samples to be almost flat, with little structural variation. For these reasons, we have ignored the effects of surface morphology here.

\section{Hydrophobic Nature of Regenerated Cellulose}

Structural anisotropy of cellulose may provide regenerated cellulose with the opposite aspect to the above, namely hydrophobic properties. The hydrophobic nature of cellulose can be deduced from the fol- lowing experimental facts. Silicone oil and hydrocarbons are easily dispersed in water ${ }^{4}$ in the presence of a small amount of cellulose. Hydrophobic solvents such as hexane, toluene and dichloromethane remain captured in cellulose domains even after rigorous vacuum drying. ${ }^{3}$ Some relaxation peaks of cellulose in thermally stimulated depolarized current measurements shift to lower temperature in nonpolar solvents such as hexane or benzene. ${ }^{5}$ These suggest that there must exist hydrophobic domains in cellulose.

Figure 6 is a schematic representation of the (110) 
Table II. Surface energies of crystal faces

\begin{tabular}{lccr}
\hline & \multicolumn{3}{c}{ Surface Energy $/ \mathrm{mN} \mathrm{m}^{-1}$} \\
\cline { 2 - 4 } Polymorphs & $\mathrm{d}_{1}$ & $\mathrm{~d}_{2}$ & \multicolumn{1}{c}{$\mathrm{d}_{3}$} \\
\hline Cell- $_{\alpha}$ & $154(100)$ & $137(010)$ & $62(110)$ \\
Cell- $_{\beta}$ & $155(1-10)$ & $155(110)$ & $92(200)$ \\
Cell-II & $178(1-10)$ & $101(110)$ & $110(020)$ \\
\hline
\end{tabular}

$\mathrm{d}_{1}, \mathrm{~d}_{2}, \mathrm{~d}_{3}$ : arbitrary symbols of crystal faces ranged by their wider spacings. ( ): crystal indices.

plane of cellulose II crystals. The (110) plane is quite different from the (1-10) plane because there are almost no hydroxyl groups on the (110) plane but rather, hydrogen atoms of $\mathrm{C}-\mathrm{H}$ bonds. This also helps explain the hydrophobic nature and low surface energy of the (110) plane. In order to determine the contact angle and the surface energy of this hydrophobic surface, we attempted to prepare a film with the (110) plane-oriented parallel to the film surface. Because of experimental difficulties in making this film, we estimated the surface energies of each crystal face by computer simulation.

Table II shows the surface energies of the crystal faces of various cellulose polymorphs. Symbols $d_{1}$, $\mathrm{d}_{2}$, and $\mathrm{d}_{3}$ are arbitrary and indicate crystal faces arranged by their wider spacings. The numerals in parentheses denote crystal indices. The crystal face with the highest surface energy was (1-10) of Cell II, with a value of $178 \mathrm{mN} \mathrm{m}^{-1}$. This is consistent with the fact that regenerated cellulose films having their (1-10) planes oriented almost parallel to the film surface have the most wettable surfaces of widely used polymers, being more wettable than PVA and starch, as described before. The surface energy of the (110) plane of Cell II, $101 \mathrm{mN} \mathrm{m}^{-1}$, was much less than that of (1-10), supporting the expectation that the (110) plane of Cell II is hydrophobic. If the (110) plane of Cell II were oriented parallel to the film surface, the resulting cellulose would be hydrophobic. It is noteworthy that the surface energies of hydrophobic planes of cellulose I polymorphs were quite low. In particular, the value of $62 \mathrm{mN} \mathrm{m}^{-1}$ for Cell $\mathrm{I}_{\alpha}$ was only one third of the value for the (1-10) plane of Cell II, and lower than $92 \mathrm{mN} \mathrm{m}^{-1}$ found for Cell $\mathrm{I}_{\beta}$. Cellulose is in contact with many substances such as lignin, proteins and other polysaccharides found in wood. The difference between the surface energies of Cell $\mathrm{I}_{\alpha}$ and Cell $\mathrm{I}_{\beta}$ may result from various interactions of cellulose with these substances during crystallization or initial periods of structural formation of cellulose.

As mentioned above, regenerated cellulose generally has a uniplanar orientation of (1-10) planes parallel to the film surface, providing remarkable hydrophilic properties. This orientation can be explained by the following hypothetical mechanism for the formation of the structure of cellulose regenerated from solution. Glucopyranose rings are stacked with each other by hydrophobic interactions in order to reduce hydrophobic surface area in highly polar solvents such as aqueous media, providing thermodynamic stability. The stacks form themselves into molecular sheets related to the (1-10) plane, as shown in Figure 5, which depicts a hydrophilic surface (because of the many hydroxyl groups). Volume contraction of coagulating cellulose solutions, shrinking perpendicular to the film surface, gives rise to molecular sheets with anisotropic shapes oriented parallel to the film surface, followed by the formation of crystalline and amorphous regions by aggregation of the molecular sheets by hydrogen bonding. As a result, hydrophilic film surfaces with uniplanar orientation of (1-10) planes inevitably emerge in highly polar media through the formation of molecular sheets because of thermodynamic constraints. This hypothesis is supported by the following facts. Coagulation systems with pronounced volume contraction give high $\mathrm{f}(1-10)$ values. ${ }^{19}$ The use of nonpolar solvents (e.g., toluene) as coagulants prevents the formation of molecular sheets, giving low $\mathrm{f}(1-10)$ and low crystallinity. The existence of the molecular sheet structures necessary for this hypothesis is also supported by Hayashi ${ }^{20}$ and Hermans, ${ }^{21}$ who identified the structures (naming them Plane Lattice Structures or Sheet-like Structures) as the basic and dominant features of regenerated cellulose.

As mentioned before, if we could make (110) planes oriented parallel to the film surface, hydrophobic cellulose could be prepared. According to our hypothesis, cellulose dissolution in a nonpolar solvent followed by the formation of (110) molecular sheets by hydrogen bonding, instead of (1-10) planes formed by hydrophobic interaction, could provide such a hydrophobic structure. This is quite difficult because only highly polar solvents which interact strongly with the hydroxyl groups of cellulose can actually dissolve cellulose.

\section{Changes in Wettability by Post-Treatments}

Because of the difficulties in obtaining hydrophobic surfaces from cellulose/polar solvents systems, we tried to control wettability by post-treatments. Cellophane, whose $\mathrm{f}(1-10)$ is 0.6 and water contact angle is $11.6^{\circ}$, as shown in Table I, was subjected to several post-treatments such as exposure to cyclohexane, liquid ammonia and hot glycerin. Although cellulose II polymorphs remained unchanged by the cyclohexane treatment, liquid ammonia and hot glycerin treatment transformed cellulose II into cellulose III and cellulose IV, respectively. Table III shows changes in water contact angles resulting from the post-treatments. 
Table III. Effect of post-treatments on contact angle

\begin{tabular}{llc}
\hline Crystal form & Treatment & $\begin{array}{c}\text { Contact angle } \\
/ \text { deg. }\end{array}$ \\
\hline Cell II & cyclo-hexane & 14.6 \\
Cell III & liquid ammonia & 39.6 \\
Cell IV & high temp. glycerol & 24.0 \\
\hline
\end{tabular}

The cyclohexane treatment slightly increased the contact angle to $14.6^{\circ}$ and decreased wettability, while leaving $\mathrm{f}(1-10)$ almost unchanged. The relaxation peak at $50^{\circ} \mathrm{C}$ in Thermally Stimulated Depolarized Current analysis in the dry state shifted to lower temperature $\left(-10^{\circ} \mathrm{C}\right)$ in cyclohexane. ${ }^{5}$ This means that some cellulose molecules or structural domains were mobile at room temperature in the presence of cyclohexane. Under these conditions, hydroxyl groups could possibly move to the interior of the structure from the surface, then hydrophobic planes of glucopyranose rings come to the surface.

Liquid ammonia treatment converted Cell II to Cell III and increased water contact angle remarkably, to $39.6^{\circ}$. This decrease in wettability must be related to the fact that wrinkles and shrinkage in cellulose fabrics during washing can be effectively prevented by liquid ammonia treatment. This must also be related to the reduction of complement activity from 90 to 50, imparting biocompatibility to artificial kidneys made from cellulose. Complement activity, resulting from the reaction between thioester groups in the complements in blood and hydroxyl groups on the cellulose surface, is thus affected by wettability.

The contact angle increased to $24.0^{\circ}$ after hot glycerol treatment, which changed Cell II to Cell IV. Many experimental phenomena related to hot glycerol treatments, e.g., increase in wet strength, decrease in water swelling of cuprammonium rayon and stable crystal structure (Cell IV) in water, may be related to the decrease in wettability.

The decrease in wettability by the liquid ammonia and hot glycerin treatments may result from low dielectric constants of these liquids, not from the crystal transitions. The low dielectric constants of liquid ammonia (25) and glycerol (42.5), both much lower than that of water (80), possibly cause a change in the direction of surface hydroxyl groups, resulting in lower wettability.

We have found the following three key factors to affect the wettability of cellulose. Cellulose molecules have two inherently different structural moieties, the hydrophilic and hydrophobic parts, located at the equatorial and axial positions of glucopyranose rings, respectively. Structural anisotropy of cellulose crystals derived from the above-mentioned molecular anisotropy gives rise to hydrophilic and hydrophobic crystal planes, whose surface energies are calculated to be quite different. Planar orientation of hydrophilic planes parallel to the film surface gives cellulose one of the most hydrophilic surfaces of known polymers.

\section{REFERENCES}

1. "Kagakubinran Kisohen II" 3rd ed, The Chemical Society of Japan, Ed., 1984, p 90.

2. T. Matsunaga and Y. Ikada, in "Modification of Polymers," C. E. Carraher, Jr. and M. Tsuda, Ed., ACS Symposium Series, No. 121, 1980, pp 391-406.

3. for example, H. Staudinger, K.-H. In der Birken, and M. Staudinger, Makromol. Chem., 9, 148 (1953).

4. H. Ono, Y. Shimaya, T. Hongo, and C. Yamane, Materials Research Society of Japan, 26, 569 (2001).

5. C. Yamane, M. Mori, and K. Okajima, Polym. J., 28, 1039 (1996).

6. R. Fujioka and S. Manabe, '99 Pusan-Kyeongnam/KyushuSeibu Joint Symposium on High Polymers (9th) and Fibers (7th), Gyeongszng National University, Chinju, Korea, November 2, Proceedings, P102-105 (1999).

7. W. Gibbson, L. Spencer, and R. McCall, J. Chem. Soc., 117, 479 (1920).

8. W. Brown and R. Wikström, Eur. Polym. J., 1, 1 (1966).

9. A. F. Turbak, "Proceedings 1983 International Dissolving and Specialty Pulps Conference," Tappi, Atlanta, U.S.A., 1983, p 105.

10. a) T. Takahashi, Sen-I Gakkaishi, 25, 80 (1969).

b) T. Takahashi, Sen-I Gakkaishi, 25, 122 (1969).

11. R. N. Wenzel, J. Phys. Colloid Chem., 53, 1466 (1949).

12. T. Matsunaga, Y. Ikada, and R. Kitamaru, Polym. Prepr., Jpn., 27, 475 (1978).

13. B. R. Ray, J. R. Anderson, and J. J. Scholz, J. Phys. Chem., 62, 1220 (1958).

14. Y. Nishiyama, J. Sugiyama, H. Chanzy, and P. Langan, J. Am. Chem. Soc., 125, 14300 (2003).

15. Y. Nishiyama, P. Langan, and H. Chanzy, J. Am. Chem. Soc., 124, 9074 (2002).

16. P. Langan, Y. Nishiyama, and H. Chanzy, Biomacromolecules, 2, 410 (2001).

17. S. Manabe, M. Iwata, and K. Kamide, Polym. J., 18, 1 (1986).

18. A. Isogai, in "Seruroosu no Zairyoukagaku (Material Science of Cellulose)," Toyo U. Ed., 2001, p 2.

19. T. Hongo, C. Yamane, M. Saito, and K. Okajima, Polym. J., 28, 769 (1996).

20. J. Hayashi, J. Masuda, and Y. Watanabe, Nippon Kagaku Kaishi, 5, 948 (1974).

21. P. H. Hermans, J. Polym. Sci., 4, 145 (1949). 